\title{
The potential role of vagus-nerve stimulation in the treatment of HIV-associated depression: a review of literature
}

This article was published in the following Dove Press journal:

Neuropsychiatric Disease and Treatment

28 June 2017

Number of times this article has been viewed

\section{William C Nicholson \\ Mirjam-Colette Kempf \\ Linda Moneyham \\ David E Vance}

School of Nursing, University of Alabama at Birmingham, Birmingham, AL, USA
Correspondence: William C Nicholson School of Nursing, University of Alabama at Birmingham, Room 328B, I70 I University Boulevard, Birmingham, AL 35249-1210, USA

$\mathrm{Tel}+\mid 205996982$ |

Email chancen@uab.edu
Abstract: Depression is the most common comorbidity and neuropsychiatric complication in HIV. Estimates suggest that the prevalence rate for depression among HIV-infected individuals is three times that of the general population. The association between HIV and clinical depression is complex; however, chronic activation of inflammatory mechanisms, which disrupt central nervous system (CNS) function, may contribute to this association. Disruptions in CNS function can result in cognitive disorders, social withdrawal, fatigue, apathy, psychomotor impairment, and sleep disturbances, which are common manifestations in depression and HIV alike. Interestingly, the parasympathetic system-associated vagus nerve (VN) has primary homeostatic properties that restore CNS function following a stress or inflammatory response. Unfortunately, about $30 \%$ of adults with HIV are resistant to standard psychotherapeutic and psychopharmacological treatments for depression, thus suggesting the need for alternative treatment approaches. VN stimulation (VNS) and its benefits as a treatment for depression have been well documented, but remain unexplored in the HIV population. Historically, VNS has been delivered using a surgically implanted device; however, transcutanous VNS (tVNS) with nonsurgical auricular technology is now available. Although it currently lacks Food and Drug Administration approval in the US, evidence suggests several advantages of tVNS, including a reduced side-effect profile when compared to standard treatments and comparable results to implantable VNS in treating depression. Therefore, tVNS could offer an alternative for managing depression in HIV via regulating CNS function; moreover, tVNS may be useful for treatment of other symptoms common in HIV. From this, implications for nursing research and practice are provided.

Keywords: tVNS, depression, HIV, vagus nerve

\section{Introduction}

Depression is the most common comorbidity and neuropsychiatric complication in HIV-positive adults, with an occurrence rate of approximately $40 \%-60 \%$ over a lifetime. Moreover, estimates suggest that the prevalence rate for depression among HIV-infected individuals is three to four times that of the general population. ${ }^{1-3}$ Approximately $55 \%-65 \%$ of adults with HIV treated for depression experience a reduction in depressive symptoms. ${ }^{4}$ However, as many as $30 \%$ of adults with HIVassociated depression are resistant to standard treatments (eg, psychopharmacology and psychotherapy), suggesting the need for alternative treatment approaches. As such, novel technologies should be explored for use in this population.

The association between HIV and clinical depression is complex. Chronic activation of stress mechanisms that disrupt central nervous system (CNS) function likely drives 
this association, as both conditions present with pathological proinflammatory states. According to Kim et al, ${ }^{5}$ a significant relationship exists between depression and increases in proinflammatory cytokines (such as IL6, IL1 $\beta$, TNF $\alpha$, and CRP); moreover, severity of depression is correlated with reductions in BDNF. Likewise, proinflammatory cytokines and reduced BDNF have been associated with the progression of HIV and its associated neurocognitive disorders. ${ }^{6,7}$

Comorbid depression in HIV reduces antiretroviral medication adherence, which independently leads to poor disease outcomes related to inflammation; however, depression is also independently and adversely associated with HIV progression via decreased $\mathrm{CD}^{+}$and $\mathrm{CD} 8^{+} \mathrm{T}$ lymphocytes, along with increased viral loads. ${ }^{1,8}$

In both disorders, the dysregulation of neuroendocrine and neuroimmunological systems (via proinflammatory cytokines) produces a cascade of events resulting in attenuation of parasympathetic tone, pain, psychomotor disturbance, depression, alterations in mood and cognition, anhedonia, fatigue, apathy, cognitive disorders, dysregulated sleep, appetite disruptions, and social withdrawal. These symptoms are collectively referred to as "sickness behavior", and are mediated by the vagus nerve (VN), along with being among the most prevalent symptoms in HIV with comorbid depression. ${ }^{9-12}$ Chronic disruption of the $\mathrm{VN}$ in response to stress leads to alterations in homeostasis via constant activation of the hypothalamic-pituitary-adrenal (HPA) axis and the sympathomedullary system. ${ }^{13}$ This activation increases cytokine production, and in turn induces a chronic "sickness state". Contrarily, stimulation of the VN (particularly its $\alpha_{7}$ nicotinic pathway) can reverse the effects of sickness behavior by producing an anti-inflammatory effect via regulation of cytokine expression. ${ }^{14}$ In this context, HIV and depression could be pathologically related via their effect on the $\mathrm{VN}$, and interventions targeting it could provide clinical benefit.

Implantable VN stimulation (iVNS) and its benefits in treatment-resistant depression have been well documented. ${ }^{15-18}$ iVNS is achieved via a surgically implanted device; however, transcutaneous VNS (tVNS) with nonsurgical auricular technology is now available and offers potential advantages, due to its comparable benefits to VNS, ease of use, higher accessibility, and reduced side-effect profile, ${ }^{19}$ yet the effectiveness of these technologies remains unexplored in HIV.

The main purpose of this review is to explore tVNS's potential use for depression in the HIV-infected population. First, we provide a basic overview of the VN's association to HIV and depression. Second, we briefly describe tVNS.
Third, the clinical efficacy of iVNS in depression is discussed. Fourth, studies evaluating tVNS's effectiveness in the treatment of depression since its introduction in 2010 are reviewed. Finally, implications for research and clinical practice are provided.

\section{The vagus nerve's inflammatory association with HIV and depression}

A comprehensive description of the inflammatory response is beyond the scope of this review (see Del Guerra et $\mathrm{al}^{20}$ for a more thorough review). However, in order to understand the association between depression and HIV, a brief and basic overview of their relationship to inflammation in the context of the VN is described. Psychological stress (eg, stigmatism related to HIV diagnosis, discrimination), recurrent stressors, resilience, and the inflammatory properties of HIV are likely synergistic with the chronic activation of immune cells and resultant depression. As such, HIV and depression will be referred to as HIV-associated depression for simplicity and to indicate their relationship via immunodisruption. This section focuses on HIV as being the primary stressor that contributes to depression. Specifically, discussion is focused on sickness behavior in the context of HIV-associated depression, followed by associations with the VN.

\section{Sickness behavior in HIV-associated depression}

HIV activates T cells and monocytes (eg, $\mathrm{CD} 4^{+}$), which enter the CNS. Upon entry, these activated cells infect microglia and astrocytes, while also inducing the production of proinflammatory cytokines. This cell activation (together with perivascular macrophages) causes the release of neuroexcitatory amino acids (eg, glutamate), thereby disrupting calcium channels and propagating an inflammatory state. Additionally, cell activation promotes the formation and release of nonstructural proteins such as HIV-Tat and gp120. As a result, the blood-brain barrier is weakened and becomes vulnerable to peripheral cytokines, which further perpetuates the inflammatory cycle and disrupts neuronal integrity. ${ }^{21,22}$

As a result, locus coeruleus-norepinephrine projections to the hypothalamus and brain stem are activated. In response, the amygdala and HPA axis stimulate the release of glucocorticoids (cortisol) and activate the sympathomedullary system, which releases catecholamines (noradrenaline and adrenaline) into the peripheral blood. This activation coordinates glucocorticoid and catecholamine interaction with immune cells, thereby promoting cytokine expression and their transcription factors. Importantly, while antiretrovirals 
buffer this cytokine expression, they do not completely reverse this response..$^{20,23}$

Consequently, unsuppressed cytokine release activates an inflammatory state that manifests as "sickness or depressive behavior". Under normal conditions, this behavior is protective, and regresses as cytokines normalize along with the inflammatory response. The rebalancing of cytokines allows them to resume their trophic responsibilities in promoting neurogenesis, neuroplasticity, and maintenance of cognitive function. ${ }^{24}$ However, in the case of HIV, this sickness behavior persists into a pathological inflammatory state. Importantly, the $\mathrm{VN}$ is grossly responsible for initiating sickness behavior via its interaction with cytokines, and is a critical response to immunoactivation.

\section{Vagus nerve and sickness behavior}

Herz and Kipnis ${ }^{25}$ suggested the VN mediates sickness behavior. The VN is a major component of the autonomic nervous system, which influences neuronal, endocrine, and immune functions (neuroendocrine-immune axis) via its efferent (motor) and afferent (sensory) pathways. The vagal cholinergic anti-inflammatory pathway (CAP) interacts with the CNS, and is primarily responsible for sickness behavior.

During acute-stress states, the CAP is activated in the presence of a stressor and is responsive to proinflammatory signals that initiate an immune response. Vagal afferents detect central or peripheral pathogens (eg, via Toll-like receptors) and carry proinflammatory cytokine signals to the brain-stem nuclei. These signals are converted to the nucleus tractus solitarius and project to autonomic output centers (eg, limbic system, hippocampus, frontal cortex). The medulla coordinates this response and stimulates vagal efferent fibers, which project to the celiac ganglion. ${ }^{26}$ Vagal fibers develop synapses on cell bodies within the celiac ganglion, thus activating innate immune responses in the spleen. Splenic nerves then release norepinephrine, which communicates with $\beta_{2}$-adrenergic receptor-expressing $\mathrm{T}$ cells. Consequently, $\mathrm{T}$ cells release acetylcholine to interact with $\alpha_{7}$ nicotinic receptors, which are present on the cell surfaces of macrophages and other inflammatory cells (eg, monocytes). ${ }^{27}$ Complex, vagal-associated brain activity and signaling inhibits inflammation due to suppression of cytokines, thereby regulating their release and stabilizing the stress response.

Contrarily, chronic inflammation (such as is the case with HIV-associated depression) interferes with the VN's ability to regulate the immune response successfully by disrupting signaling in the $\mathrm{CAP} .{ }^{28}$ As a result, glutamate and $\gamma$-aminobutyric acid, along with central cytokine systems, are chronically disrupted (creating a persistent proinflammatory state), which interferes with their trophic and homeostatic function. $\mathrm{CD}^{+}$and $\mathrm{CD} 8^{+} \mathrm{T}$ lymphocytes are dysregulated at the splenic level, which disrupts catecholamine function, thereby perpetuating stress signaling to the CNS., ${ }^{1,23}$

This chronic increase in cytokine and stress signaling via a disrupted CAP persistently activates the amygdala, HPA axis, and sympathomedullary system, which interferes with appetite and sleep; ${ }^{11}$ moreover, this allows excessive cortisol to bind to glucocorticoids and increases insulin resistance. The resulting hypercortisolism and the actions of HIV's gp120 protein interferes with BDNF's energy metabolism, which supports brain health by lowering blood glucose, reducing insulin resistance, and regulating food intake. ${ }^{29}$ Consequently, BDNF is reduced and metabolic disruptions occur, which interfere with neuroplasticity and neurogenesis..$^{30}$ Additionally, during this dysfunctional immune response, nucleus accumbens activity is significantly downregulated, which interferes with the reward pathway (motivation, pleasure, and reinforcement learning), thus promoting anhedonia and apathy. ${ }^{31}$ Moreover, increased cytokine signaling to the basal ganglia results in psychomotor disturbances and fatigue..$^{32,33}$ The cytokine-induced CAP disruption compromises the prefrontal cortex and its functional capacity to maintain executive function, attention, memory, and adaptive social behaviors. Ultimately, this disruption perpetuates pathological social withdrawal, anhedonia, fatigue, psychomotor disturbances, and alterations to both mood and cognition or sickness behavior. ${ }^{24}$

The chronic-stress mechanisms in HIV-associated depression are complex and have not yet been fully elucidated; however, the overlapping symptomatology in HIV-associated depression symptoms and their relationship to $\mathrm{VN}$ function should be considered a potential target for treatment interventions.

\section{Vagus-nerve stimulation}

The US Food and Drug Administration approved iVNS for use in treatment-resistant depression in 2005, while tVNS was approved in Europe for depression in 2010. One such tVNS device called Nemos (developed by Cerbomed in Erlangen, Germany) has received authorization for use in epilepsy, depression, and pain conditions. Likewise, another tVNS device called GammaCore (developed by ElectroCore LLD in Basking Ridge, NJ, US) has European approval for use in prophylactic and acute treatment of migraines and headaches; however, it is not approved for depression. Currently, tVNS 
is not approved in the US, but is being used for research trials across multiple populations (eg, eating disorders, rheumatoid arthritis, and irritable bowel syndrome). ${ }^{34}$

\section{Transauricular vagus-nerve stimulation}

The tVNS unit attaches to a battery-powered control unit that connects to a headset with bilateral electrodes, which are placed on the outer ear (see Figure 1). Alternatively, adhesive anodes and cathodes can be placed over the mastoid process juxtaposed to the outer ears. Electrodes can be applied either unilaterally or bilaterally, depending on the device specifications. ${ }^{35}$

Three sensory nerves supply the outer ear (ie, auriculotemporal and great auricular), but the tVNS unit specifically targets the auricular branch of the $\mathrm{VN}(\mathrm{ABVN})$ and to a lesser degree the greater auricular nerve. The ABVN predominantly innervates the external auditory meatus and concha (cymba and cavum), which provides the cutaneous access field of the ABVN targeted by tVNS. ${ }^{34,36}$ The concha's nerve fibers provide the electrical stimulation point that allows changes in intensity, pulse duration, and frequency from the tVNS unit to induce signals via the myelinated $A \beta$ fibers of the ABVN. ${ }^{36}$ These ABVN fibers terminate in the nucleus of the solitary tract of the brain stem. Neuronal projections from this nucleus relay excitatory and inhibitory signals. These vagal projections are stated to be responsible for its neuroplastic, neurogenic, neuroprotective, and antidepressant properties. ${ }^{37}$

Stimulation can be self-administered, yet stimulation parameters have not been established; therefore, clinical models for this device do not include a standardized duration, frequency, or administration paradigm. The manufacturers recommend daily use (one to four times) for at least 1 hour (maximum of 4 hours); however, research regarding the efficacy of these specific parameters is limited. Various approaches have been researched, but are contingent on which specific tVNS unit is being utilized. ${ }^{38,39}$ In all studies, intensity is adjusted based on perceptual tolerance and kept below each individual's pain threshold. Pain thresholds are defined as the minimal amount of stimulation that evokes a tingling or unpleasant sensation.

The side-effect profile for tVNS is appealing, due to it being the least innocuous of standard biological treatments for depression. Adverse effects tend to be stimulation-related and tend to occur during those time intervals. The most common symptoms reported are itching, outer-ear discomfort, local pain on stimulation side, and neck pain that corresponds anatomically to the location of the electrodes. Additionally, VNS can be safely used in pregnancy along with concomitant psychotropic medications (eg, antidepressants) and electroconvulsive therapy. ${ }^{18}$

\section{Depression and iVNS}

Approximately 21 research studies have been conducted to examine the relationship between iVNS and treatmentresistant depression. Meta-analytic and systematic reviews have found a beneficial effect for iVNS in this population. ${ }^{40-42}$ These beneficial results support the inclusion of iVNS as a treatment option in national guidelines for depression (eg, Canadian Network for Mood and Anxiety Treatments, British

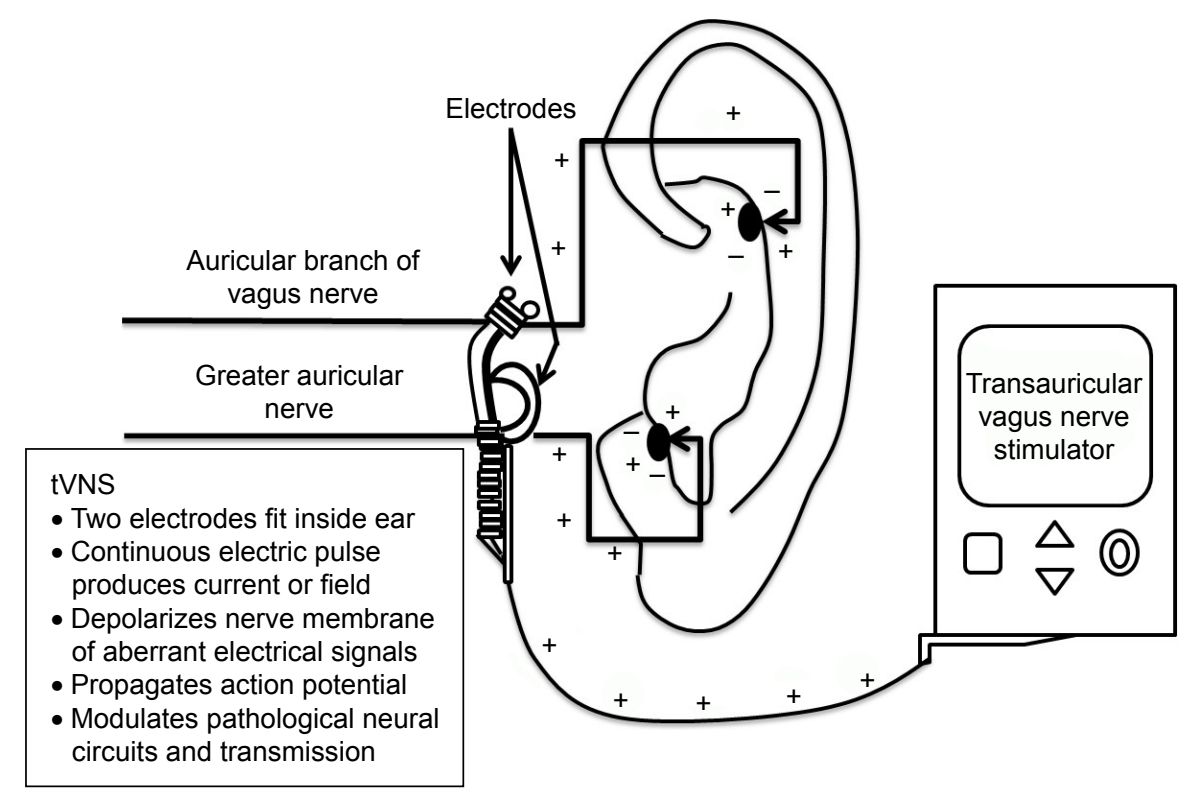

Figure I Transcutaneous (auricular) vagus nerve-stimulation (tVNS) device. 
Association for Psychopharmacology, and National Institute for Health and Care Excellence). ${ }^{41,43-45}$

However, due to the methodological limitations of iVNS (cost, surgical requirements, and the higher risk posed to participants by implanting a sham device), a majority of the iVNS studies did not utilize a control group. This thus limits the inferences that can be made about the findings. Instead, most utilized a before-after design, which increases the potential for a placebo effect and could explain a portion of beneficial results, thereby minimizing the overall effect. ${ }^{42}$

Of note, in the studies where randomized controlled trials were utilized, results were mixed. Rush et $\mathrm{al}^{46}$ did not demonstrate any significant benefits for VNS in a 10-week $(\mathrm{n}=235)$, acute, blind, randomized controlled trial. Contrarily, in a 12-month naturalistic follow-up to this study, Rush et $\mathrm{al}^{47}$ found a significant reduction in depressive scores and global improvement. Likewise, Sackheim et $\mathrm{al}^{48}$ conducted both a pilot and randomized controlled study. In the latter study, participants were randomized into active or sham groups $(n=205)$, with $15 \%$ of the early responders showing a $50 \%$ reduction in depressive symptoms at 30 months and $77 \%$ maintaining this response at 24 months. Of the late responders, $20 \%$ showed a reduction at 12 months and $65 \%$ maintained this response at 24 months.

Due to the lack of adequate control trials and mixed results of the randomized studies available, more research is needed to determine the utility of iVNS as an effective intervention for treatment-resistant depression. Fortunately, the novel tVNS devices allow researchers to bypass the limitations of traditional iVNS and enable more rigorous clinical trials. As such, it is important to review the available tVNS research to determine its efficacy for depressive symptomatology and whether the advantageous nonsurgical, transauricular design improves on the previous methodological limitations of traditional iVNS. If effective, these devices could offer those experiencing HIV and comorbid depression an alternative for treatment.

\section{Materials and methods}

A systematic PubMed search of the literature was conducted on December 1, 2016 for 2010-2016. The PRISMA (Preferred Reporting Items for Systematic Reviews and MetaAnalyses) approach (see Figure 2) was used as the method for conducting the literature review. ${ }^{49}$ The year 2010 was chosen due to the tVNS unit being made available to the general research market at that time. One study was conducted in 2007 that utilized this device. ${ }^{50}$ However, it did not directly measure the effects of tVNS on depression, and thus was not included. The search was restricted to peerreviewed research articles published in English. Keywords operated in the search were "tVNS and depression" yielding 35 articles and "tVNS and HIV" yielding 0 articles. The tVNS and depression results were narrowed by removing the words, "epilepsy", "tinnitus", and "implantable", which resulted in ten articles. Inclusion criteria further limited the review to quantitative clinical trials and human studies, which netted five articles. Due to the small number of results, case studies were added back, resulting in a total of six articles. A more detailed review of these studies can be found in Table 1.

\section{Results}

Hein et $\mathrm{al}^{38}$ conducted the first pilot study to explore the effects of tVNS in major depression $(n=37)$. Two randomized sham-controlled (add-on) studies were conducted, with participants being stimulated for 5 days each week for 14 days. The first study ( $\mathrm{n}=22)$ used an active-control group $(n=11)$ and a sham-control group. The sham control received stimulation to the center of the left earlobe, which is without vagal innervation. Stimulation intensity was 0-600 mA with a frequency of $1.5 \mathrm{~Hz}$ for 15 minutes daily. Ranges were adjusted just below the participant's perception threshold. In the second study $(n=15)$, seven participants were given active stimulation while the remaining received sham stimulation. However, the stimulation occurred for 15 minutes twice daily (morning and evening) with fixed parameters of $130 \mathrm{~mA}$ and 1.5 Hz. The Hamilton Depression Rating Scale (HAM-D) and Beck Depression Inventory (BDI) were administered at baseline (day 0) and at the conclusion of the study (day 14). Using pooled analysis, both studies showed a significant mean difference between the active and sham groups on the BDI. Contrarily, neither showed significant differences on the HAM-D. No significant side effects were noted due to tVNS use.

Trevizol et $\mathrm{al}^{35}$ reported the first case-study utilizing tVNS in a 38-year-old man with major depression. This patient received stimulation for 5 days each week for 14 days at $120 \mathrm{~Hz}$ with a pulse duration of $250 \mu$ s for 30 minutes daily. The HAM-D was administered at baseline along with the Montreal Cognitive Assessment. After 14 days, this patient's symptoms went into complete remission and remained stable at a 2-month follow-up. No significant side effects were noted due to tVNS use.

Trevizol et $a^{51}$ conducted a proof-of-concept trial $(n=12)$ exploring the effects of tVNS in major depressive disorder. The HAM-D was the primary instrument used to evaluate depression, with end-point response defined as a $50 \%$ 


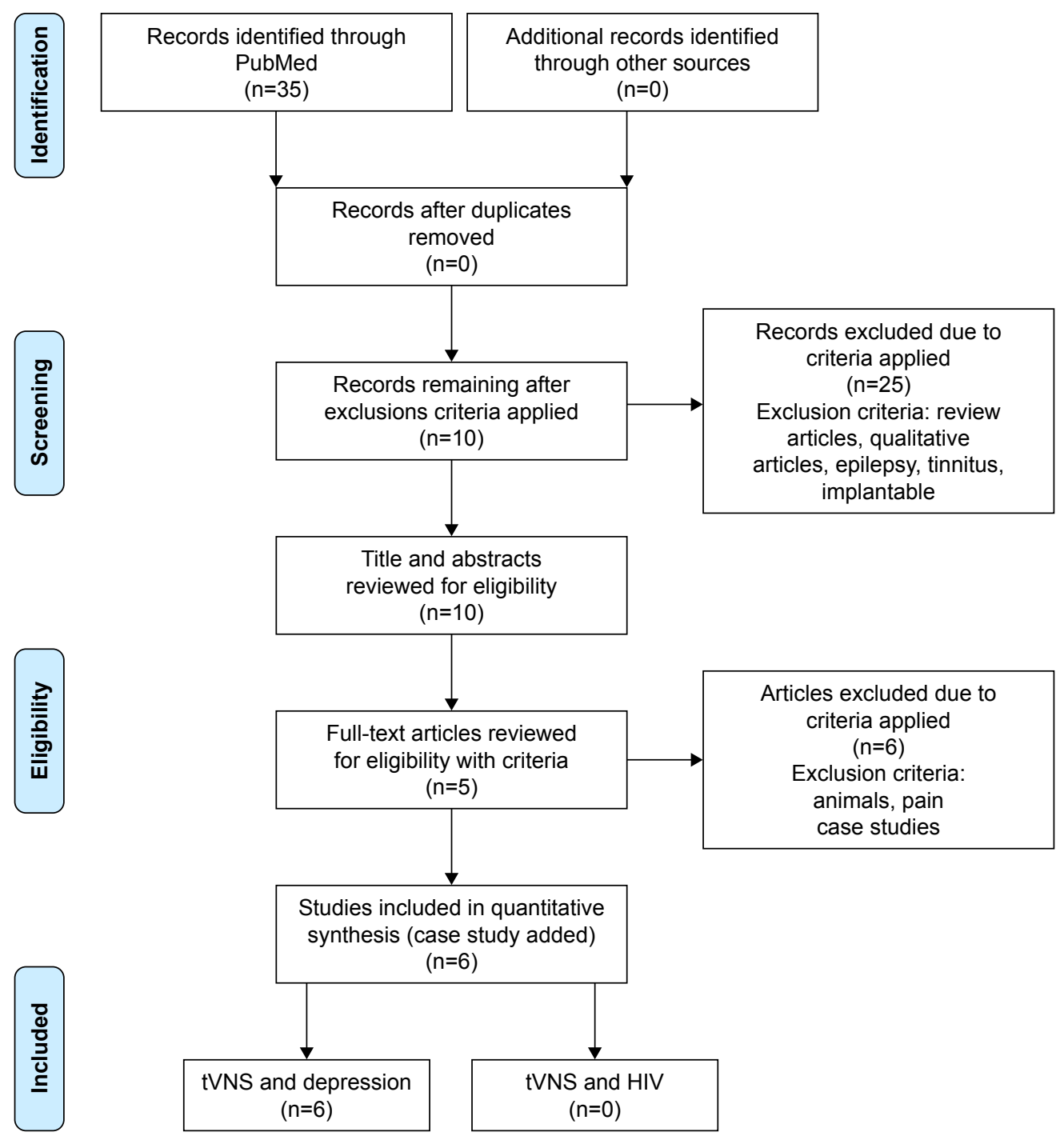

Figure 2 PRISMA flow diagram demonstrating screening method for articles.

Note: Adapted from Moher D, Liberati A, Tetzlaff J, Altman DG. Preferred reporting items for systematic reviews and meta-analyses: the PRISMA statement. PLoS Med. 2009;6(7):el000097. ${ }^{49}$

Abbreviations: PRISMA, Preferred Reporting Items for Systematic Reviews and Meta-Analyses; tVNS, transcutaneous vagus nerve-stimulation.

reduction in baseline scores. Patients received stimulation for 5 days each week for 14 days at $120 \mathrm{~Hz}$ with pulse duration of $250 \mu \mathrm{s}$, and intensity was set at $12 \mathrm{~mA}$ for 30 minutes daily. After 14 days, all participants demonstrated a clinical response with a $50 \%$ reduction in depressive symptoms. Five patients exhibited remission of depressive symptoms (score of $<8$ on the HAM-D). Response was maintained at 1-month follow-up. Cognition remained stable, as measured by the Montreal Cognitive Assessment. No adverse effects were reported. Ten participants reported diurnal sleepiness after stimulation, with six patients experiencing nausea, but no medication or treatment was required. There were no dropouts reported.

Fang et $\mathrm{al}^{52}$ conducted a single-blinded clinical trial $(n=49)$ exploring the effects of tVNS in mild-moderate major depression. A total of 34 patients were included in the study analysis (after 14 dropped out and one was excluded for excessive head movement), and were divided into active $(n=18)$ and sham $(n=16)$ groups. The active group received stimulation at $20 \mathrm{~Hz}$ with a wave width less than $1 \mathrm{~ms}$ with intensity adjusted based on tolerance or perception threshold (4-6 mA). Stimulation occurred twice a day for 30 minutes at least 5 days a week for 1 month. After functional magnetic resonance imaging (fMRI) and HAM-D to evaluate depression symptoms, the results supported the efficacy of tVNS, as significant decreases in depressive symptoms $(\leq 50 \%$ reduction) were found. In support, the fMRI results corresponded to increased functional connectivity in the default-mode network of the brain (known to be decreased in depression). No significant side effects were noted due to tVNS use. 


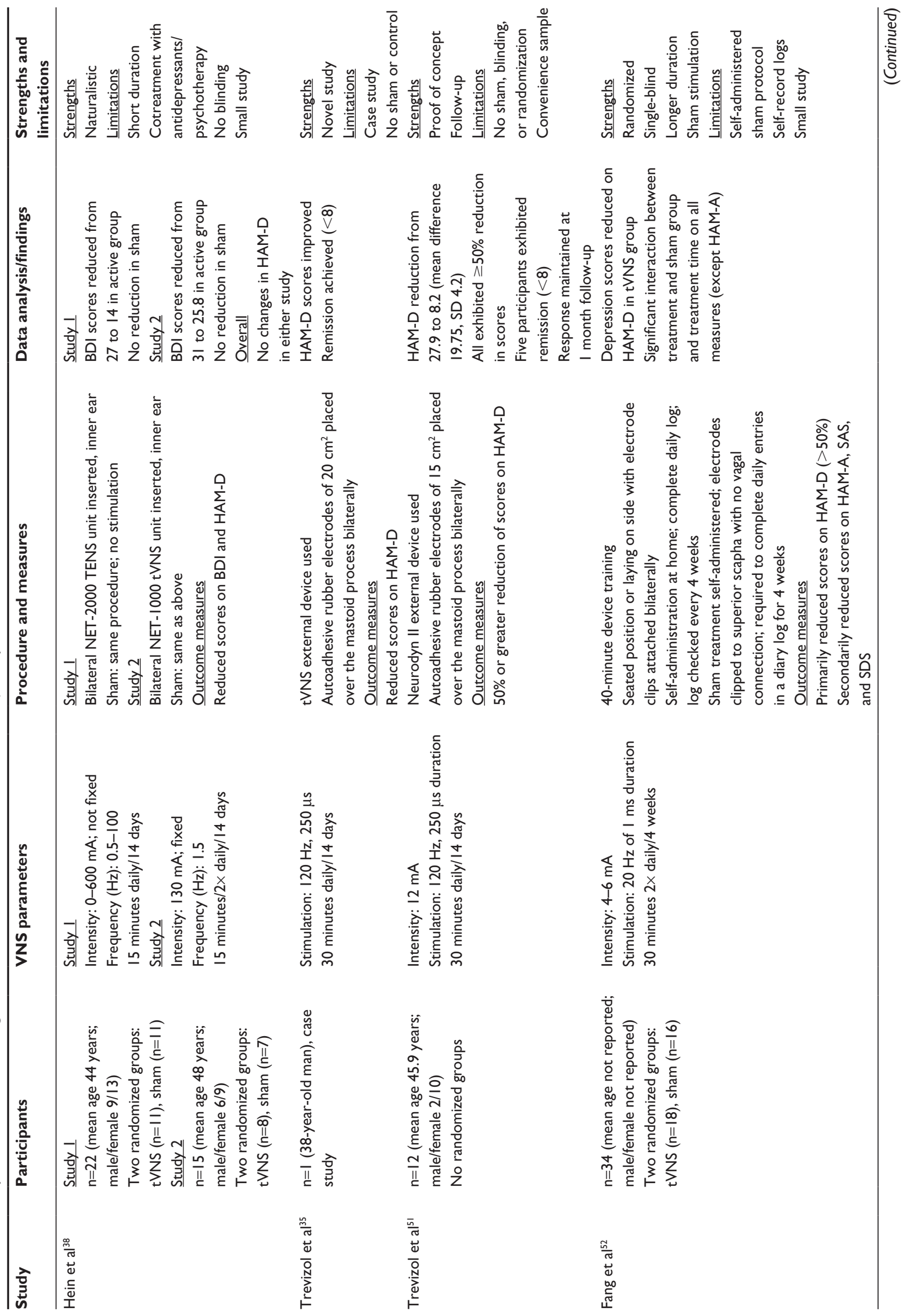




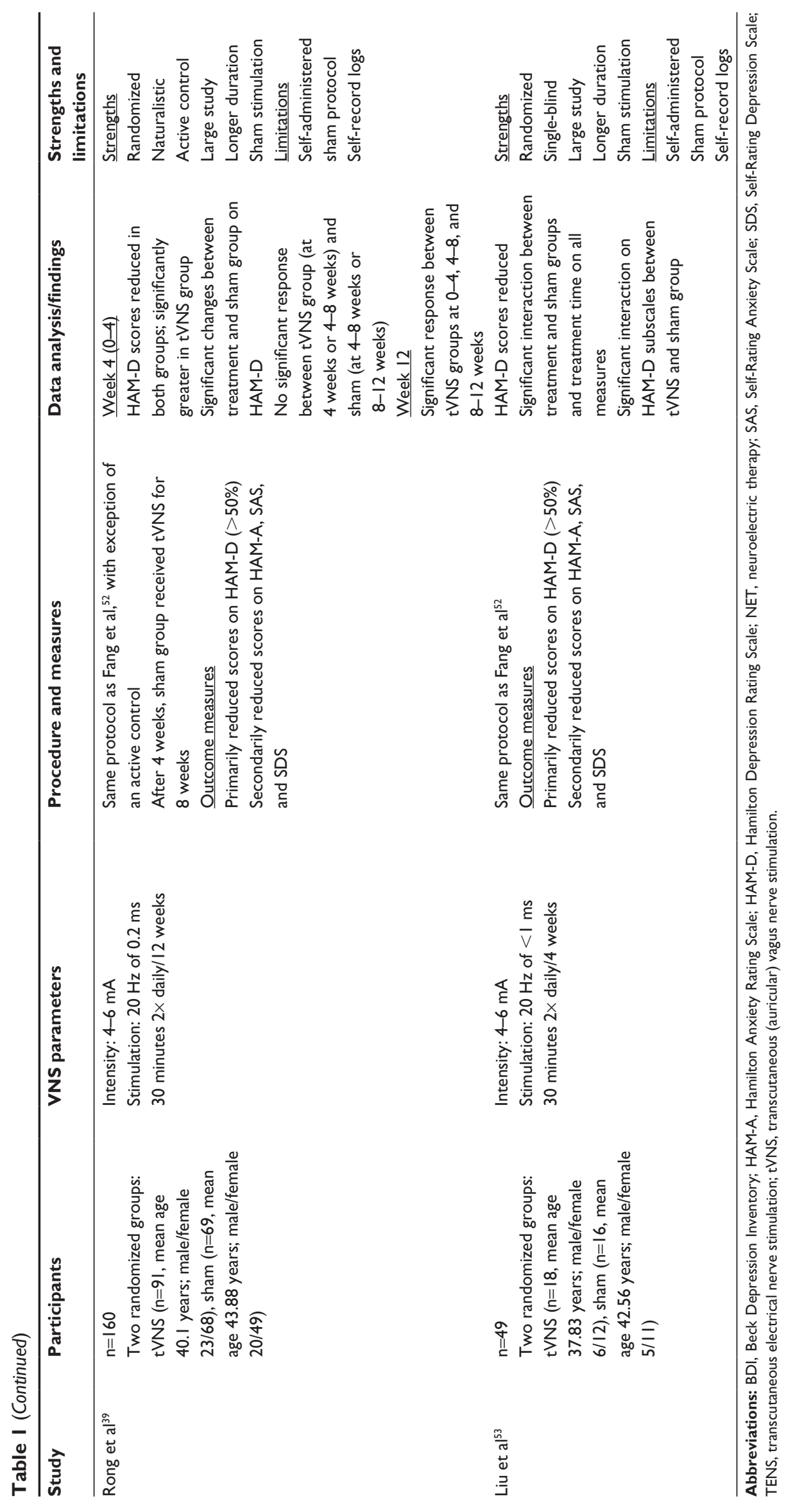


Rong et $\mathrm{al}^{39}$ conducted a nonrandomized study ( $\left.\mathrm{n}=160\right)$. The study was divided into an active group $(n=91)$ that received tVNS for 12 weeks and a control group $(n=69)$ that received sham stimulation for 8 weeks followed by 4 weeks of tVNS. A total of 148 participants completed the trial at 1 month and 138 participants at 2 months. Stimulation parameters were set at $20 \mathrm{~Hz}$ (width of $0.2 \mathrm{~ms}$ ), with intensity increased gradually from 0 until tolerance threshold was achieved (4-6 mA). As per the other studies' protocols, stimulation was administered twice daily for 30 minutes per session. Depression was assessed using the HAM-D. After 1 month of treatment, there was a significant difference between the active group and the sham group with a decrease in scores ( $\leq 50 \%$ reduction). Additionally, this improvement was maintained at 12 weeks. Interestingly, this study explored subgroups within the tVNS participants to determine if there was a difference in response when comparing mild (HAM-D score of $<20$ ) to moderate depression. Although the effect size was smaller, this study demonstrated a significant reduction in mild symptoms as well. No adverse side effects were noted due to tVNS use.

Utilizing the same experimental and stimulation procedures same as Rong et al ${ }^{139}$ and Lui et al, ${ }^{53}$ research from Fang et a $\mathrm{l}^{52}$ conducted a single-blind clinical trial $(\mathrm{n}=49)$ exploring the neurological effects of tVNS in depression. Stimulation occurred over a 1-month period. Resting fMRI scans were conducted pre- and post-tVNS intervention, while depressive symptomatology was assessed utilizing the HAM-D as the primary end point. After 1 month of treatment, HAM-D scores significantly reduced in the tVNS group when compared to the sham. Moreover, the functional connectivity between the amygdala-lateral prefrontal networks showed a significant increase. Increases in this resting-state connectivity corresponded to the HAM-D reduction in depressive symptomatology. No significant side effects were noted due to tVNS use.

\section{Discussion}

The purpose of this review was to examine tVNS's potential use in HIV-associated depression via a review of its use in the depression population. Overall, the results of the review are promising and support the findings of previous studies that utilized traditional iVNS devices. Each found a significant reduction in depressive symptoms. Furthermore, as expected, no significant side effects occurred from utilizing the tVNS units. In an effort to standardize stimulation parameters, each study relied on a similar protocol developed by Hein et al ${ }^{38}$ for experimental reference and utilized similar procedures.
This effort and replication of positive results provides the first step in developing a standardized clinical paradigm for the use of tVNS in a clinical setting for depression. Contrarily, the limited stimulation parameters (see parameters in Table 1) restrict interpretability or feasibility of use outside those parameters. In reference to HIV-associated depression, its utilization could require a different stimulation protocol that has not yet been researched.

Interpretations of positive results should be approached with caution, as there were few research studies available, which restricts any conclusive statements about tVNS's efficacy. While some were adequately powered, ${ }^{39}$ other trials were restricted to a case-study design ${ }^{35}$ or used participants from the same cohort. ${ }^{52,53}$ Additionally, due to one instrument being utilized as the outcome measure (HAM-D) in each of these studies, it cannot be ruled out that this instrument is not capturing a heterogeneous population.

Simply, contention surrounds the accuracy of HAM-D cutoff scores for remission versus active depression. In addition, it does not include specific diagnostic information, which is included in other depression-screening scales, thereby providing a more general assessment of depression. Therefore, the construct validity of the HAM-D is of concern, due to it lacking the conceptual clarity of other available measures. This limitation could restrict the HAM-D's identification of depressive symptom subtypes. ${ }^{54}$ For example, Hein et $\mathrm{al}^{38}$ found depressive symptoms remitted utilizing the HAM-D in both the sham and control groups. A similar effect was found in Fang et al. ${ }^{52}$ This finding could suggest that the improvements in depression as it relates to tVNS are not as specific in the HAM-D versus other scales; therefore, this could affect interpretability in the context of various types of depression (such as HIV-associated depression). Despite these methodological limitations, the results of this review suggest $t$ VNS should be considered as a treatment option for HIV-associated depression.

Limitations to this review were the restriction of review articles to those in English. It is possible that other research trials have been published in the non-English literature, which could have added valuable results to this review. Considering the lack of research examining tVNS and depression in humans, animal studies (which have to be considered with caution) could have provided a deeper understanding of tVNS's potential use in HIV-associated depression.

\section{Implications for HIV research}

Based on this review, the most compelling evidence for tVNS's use in HIV-associated depression was found in the 
studies conducted by both Fang et $\mathrm{al}^{52}$ and Lui et al. ${ }^{53}$ Each of these studies explored the neurological effects of tVNS on depression via brain imaging and rating scales. The results identified improved functional connectivity in the default-mode network (eg, hippocampus, amygdala, medial prefrontal cortex, and anterior cingulate), which is involved in affect, cognition (particularly prospective memory), and emotional regulation. This confirms previous studies in depression that have implicated altered default-mode network connectivity as playing a primary role in its expression. Moreover, studies have shown cytokines have a profound effect on this network, and its sensitivity to inflammation has been linked to depression and cognitive impairment. ${ }^{55}$

Evidence from the HIV CHARTER cohort study suggests depressive episodes (over a lifetime) were present in approximately $50 \%$ of cases with neurocognitive impairment. ${ }^{56}$ Interestingly, in a cross-sectional study $\left(\mathrm{n}=111,58 \mathrm{HIV}^{+}\right.$ and $53 \mathrm{HIV}^{-}$), Thomas et $\mathrm{al}^{57}$ demonstrated diminished functional connectivity in the default-mode network as playing a critical role in the neurodegenerative process of HIV or more specifically HIV-associated neurocognitive disorder (HAND). Furthermore, they demonstrated HIV's disruption of connectivity in the salience and executivecontrol networks that are responsible for working memory and emotional conflict processing.

In a recent short-term study with 30 healthy adults ( 15 women), Jacobs et al ${ }^{58}$ found tVNS improved associativememory performance when compared to a control group. These researchers' hypothesized that tVNS mediated stressrelated effects (eg, inflammation) on memory function via its interaction with $\beta$-adrenergic receptors located on afferent vagal fibers that project into the nucleus tractus solitarius. This vagal activation stimulates the locus coeruleus to release norepinephrine, thereby modulating cortical and subcortical network activity. The results suggest tVNS changes the functional pathways for learning and consolidation that favor neuroplasticity. Interestingly, functional decline in learning efficiency and memory retrieval has increased in HIV during the antiretroviral era. ${ }^{59}$

Bachis et $\mathrm{al}^{29}$ demonstrated BDNF's ability to inhibit HIV's gp120 protein, showing an inverse relationship in its expression. This suggests BDNF helps reduce microglial and astrocyte infection in the CNS via independent and anti-inflammatory properties. Therefore, they posited that increased BDNF could counteract gp120's interaction with monocytes. Interestingly, Biggio et a ${ }^{60}$ demonstrated long-term iVNS's ability to increase BDNF expression in the hippocampus and induce neuroplasticity. This effect diminished after 3 weeks without iVNS. They concluded that long-term BDNF promoted the survival and trophism of new granule cells in the hippocampus, which persisted in the presence of iVNS.

In support of this, Furmaga et a ${ }^{61}$ demonstrated iVNS promoted increased BDNF activity via phosphorylation of its primary receptor (TrkB). These researchers noted that this activity provided an antidepressant effect that was independent of those accomplished with antidepressant medications. This mechanism suggests tVNS could augment current antidepressant medications (eg, paroxetine, sertraline), thus providing an enhanced effect or offering an alternative neurological pathway for treatment. Importantly, tVNS has been shown to modify proinflammatory cytokines in murine models. ${ }^{62}$ More recently, in a healthy sample of 20 men and women, Lerman et al ${ }^{63}$ demonstrated tVNS's ability to downregulate inflammatory cytokines in the context of lipopolysaccharides (induce an inflammatory response). While tVNS has not been explored in the context of BDNF, research supports its comparability to iVNS. This suggests that $\mathrm{tVNS}$ could help provide both antiinflammatory and antidepressant effects for HIV-associated depression via increased BDNF activity and cytokine regulation. Additionally, tVNS could possibly provide a treatment option for HAND.

Not only are the results promising in these pilot studies as relates to depression, but other tVNS studies have implications in conditions that comorbidly present in HIV and depression alike. For example, Ay et al ${ }^{64}$ demonstrated in rat models that cutaneous VNS inhibited cerebral ischemia-induced immunoactivation. Furthermore, it reduced the degree of tissue injury and functional deficiencies that resulted from the cerebral event. The effectiveness in ischemia has implications for HIV and related cerebrovascular pathology. HIV patients are admitted to hospitals $60 \%$ more often for stroke, despite a reduction in overall hospitalizations in the general population. Research suggests cerebrovascular inflammation via chronic immunoactivation could play a critical role in HAND. ${ }^{21}$ Likewise, Ayerbe et a ${ }^{65}$ reported a $29 \%$ prevalence of depression up to 10 years poststroke, with a $40 \%-52 \%$ cumulative incidence within 5 years. Importantly, predictors of this depression were prestroke depression and cognitive impairment.

Currently, no studies exist that explore tVNS as a treatment option for HIV; furthermore, no infectious models exist in nonhumans that can actually duplicate the physiological effects HIV has on humans, which suggests the need to test it directly in adults with HIV. ${ }^{66}$ The safety, effectiveness, 
and tolerability of tVNS have been reviewed for sepsis, atrial fibrillation, depression, epilepsy, migraines, and tinnitus, which provides a strong foundation for its use in HIV. In consideration of current knowledge and available technology, research in HIV-associated depression is warranted with tVNS. The potential benefits of this research are not restricted to depression or cognitive disorders, but could also be utilized as a buffer to comorbidities associated with HIV (eg, stroke, metabolic disruptions, and neuropathy) or that result as a consequence of HIV treatment.

\section{Implications for clinicians}

The safety, efficacy, and self-administration capabilities of the tVNS units have clinical utility for clinicians. If research determines their efficacy in the HIV population, these devices could provide a cost-effective and safe alternative for HIVassociated depression treatment. In an outpatient setting, these devices can be calibrated, prescribed for use at home, and utilized as augmenting agents to standard antidepressant treatments or individual therapies for comorbid depression and HIV symptomatology (eg, cognitive disorders). If effective, these devices could help reduce the polypharmacy often faced in HIV by replacing select psychotropic medications and select cardiovascular medications; ${ }^{67}$ moreover, they could potentially alleviate or help control common side effects that result from the use of these medications (eg, QRS elongation, tinnitus, headaches, metabolic disturbances, and motor dysfunction). ${ }^{68-70}$

\section{Disclosure}

The authors report no conflicts of interest in this work.

\section{References}

1. Arseniou S, Arvaniti A, Samakouri M. HIV infection and depression. Psychiatry Clin Neurosci. 2013;68(2):96-109.

2. Everall I, Vaida F, Khanlou N, et al. Cliniconeuropathologic correlates of human immunodeficiency virus in the era of antiretroviral therapy. J Neurovirol. 2009;15(5-6):360-370.

3. Nanni MG, Caruso R, Mitchell AJ, Meggiolaro E, Grassi L. Depression in HIV infected patients: a review. Curr Psychiatry Rep. 2014;17(1):530.

4. Sherr L, Clucas C, Harding R, Sibley E, Catalan J. HIV and depression: a systematic review of interventions. Psychol Health Med. 2011; 16(5):493-527.

5. Kim YK, Na KS, Myint AM, Leonard BE. The role of pro-inflammatory cytokines in neuroinflammation, neurogenesis and the neuroendocrine system in major depression. Prog Neuropsychopharmacol Biol Psychiatry. 2016;64:277-284.

6. Fields J, Dumaop W, Langford TD, Rockenstein E, Masliah E. Role of neurotrophic factor alterations in the neurodegenerative process in HIV associated neurocognitive disorders. J Neuroimmune Pharmacol. 2014;9(2):102-116.

7. Lotrich FE, Albusaysi S, Ferrell RE. Brain-derived neurotrophic factor serum levels and genotype: association with depression during interferon- $\alpha$ treatment. Neuropsychopharmacology. 2012;38(6):985-995.
8. Sin NL, Dimatteo MR. Depression treatment enhances adherence to antiretroviral therapy: a meta-analysis. Ann Behav Med. 2013;47(3): 259-269.

9. Andréasson A, Arborelius L, Erlanson-Albertsson C, Lekander M. A putative role for cytokines in the impaired appetite in depression. Brain Behav Immun. 2007;21(2):147-152.

10. Dantzer R, O’Connor JC, Freund GG, Johnson RW, Kelley KW. From inflammation to sickness and depression: when the immune system subjugates the brain. Nat Rev Neurosci. 2008;9(1):46-56.

11. Imeri L, Opp MR. How (and why) the immune system makes us sleep. Nat Rev Neurosci. 2009;10(3):199-210.

12. Wolff CL, Alvarado MR, Wolff RM. [Depression in HIV infection: prevalence, risk factors and management]. Rev Chilena Infectol. 2010; 27(1):65-74. Spanish.

13. Thrivikraman K, Zejnelovic F, Bonsall RW, Owens MJ. Neuroendocrine homeostasis after vagus nerve stimulation in rats. Psychoneuroendocrinology. 2013;38(7):1067-1077.

14. Bonaz B, Sinniger V, Pellissier S. Anti-inflammatory properties of the vagus nerve: potential therapeutic implications of vagus nerve stimulation. J Physiol. 2016;594(20):5781-5790.

15. Albert U, Maina G, Aguglia A, et al. Vagus nerve stimulation for treatment-resistant mood disorders: a long-term naturalistic study. $B M C$ Psychiatry. 2015;15:64.

16. Bajbouj M, Merk1 A, Schlaepfer TE, et al. Two-year outcome of vagus nerve stimulation in treatment-resistant depression. J Clin Psychopharmacol. 2010;30(3):273-281.

17. George MS, Rush AJ, Marangell LB, et al. A one-year comparison of vagus nerve stimulation with treatment as usual for treatment-resistant depression. Biol Psychiatry. 2005;58(5):364-373.

18. Sackheim HA, Keilp JG, Rush J, et al. The effects of vagus nerve stimulation on cognitive performance in patients with treatment-resistant depression. Neuropsychiatry Neuropsychol Behav Neurol. 2001;14(1):53-62.

19. Ben-Menachem E, Revesz D, Simon BJ, Silberstein S. Surgically implanted and non-invasive vagus nerve stimulation: a review of efficacy, safety and tolerability. Eur J Neurol. 2015;22(9):1260-1268.

20. Del Guerra FB, Fonseca JL, Figueiredo VM, Ziff EB, Konkiewitz EC. Human immunodeficiency virus-associated depression: contributions of immuno-inflammatory, monoaminergic, neurodegenerative, and neurotrophic pathways. J Neurovirol. 2013;19(4):314-327.

21. Hong S, Banks WA. Role of the immune system in HIV-associated neuroinflammation and neurocognitive implications. Brain Behav Immun. $2015 ; 45: 1-12$.

22. Saylor D, Dickens AM, Sacktor N, et al. HIV-associated neurocognitive disorder: pathogenesis and prospects for treatment. Nat Rev Neurol. 2016;12(4):234-248.

23. Rivera-Rivera Y, Vázquez-Santiago FJ, Albino E, Sánchez MD, RiveraAmill V. Impact of depression and inflammation on the progression of HIV disease. J Clin Cell Immunol. 2016;7(3):423.

24. Raedler TJ. Inflammatory mechanisms in major depressive disorder. Curr Opin Psychiatry. 2011;24(6):519-525.

25. Herz J, Kipnis J. Bugs and brain: how infection makes you feel blue. Immunity. 2016;44(4):718-720.

26. Cuoco JA, Fennie CN, Cheriyan GK. The cholinergic anti-inflammatory pathway: a novel paradigm for translational research in neuroimmunology. J Neurol Neurosci. 2016;7(2):86.

27. Ballester LY, Capó-Vélez CM, García-Beltrán WF, et al. Up-regulation of the neuronal nicotinic receptor $\alpha 7$ by HIV glycoprotein 120: potential implications for HIV-associated neurocognitive disorder. J Biol Chem. 2012;287(5):3079-3086.

28. Delgado-Vélez M, Báez-Pagán CA, Gerena Y, et al. The $\alpha 7$-nicotinic receptor is upregulated in immune cells from HIV-seropositive women: consequences to the cholinergic anti-inflammatory response. Clin Transl Immunology. 2015;4(12):e53.

29. Bachis A, Major EO, Mocchetti I. Brain-derived neurotrophic factor inhibits human immunodeficiency virus-1/gp120-mediated cerebellar granule cell death by preventing gp120 internalization. J Neurosci. 2003;23(13):5715-5722. 
30. Calabrese F, Rossetti AC, Racagni G, Gass P, Riva MA, Molteni R. Brain-derived neurotrophic factor: a bridge between inflammation and neuroplasticity. Front Cell Neurosci. 2014;8:430.

31. Gold PW. The organization of the stress system and its dysregulation in depressive illness. Mol Psychiatry. 2014;20(1):32-47.

32. Capuron L, Pagnoni G, Drake DF, et al. Dopaminergic mechanisms of reduced basal ganglia responses to hedonic reward during interferon alfa administration. Arch Gen Psychiatry. 2012;69(10):1044-1053.

33. Brydon L, Harrison NA, Walker C, Steptoe A, Critchley HD. Peripheral inflammation is associated with altered substantia nigra activity and psychomotor slowing in humans. Biol Psychiatry. 2008;63(11): 1022-1029.

34. Howland RH. Vagus nerve stimulation. Curr Behav Neurosci Rep. 2014; 1(2):64-73.

35. Trevizol AP, Taiar I, Barros MD, Liquidatto B, Cordeiro Q, Shiozawa P. Transcutaneous vagus nerve stimulation (tVNS) protocol for the treatment of major depressive disorder: a case study assessing the auricular branch of the vagus nerve. Epilepsy Behav. 2015;53:166-167.

36. Ellrich J. Transcutaneous vagus nerve stimulation. Eur Neurol Rev. 2011;6(4):262-264.

37. Grimonprez A, Raedt R, Baeken C, Boon P, Vonck K. The antidepressant mechanism of action of vagus nerve stimulation: evidence from preclinical studies. Neurosci Biobehav Rev. 2015;56:26-34.

38. Hein E, Nowak M, Kiess O, et al. Auricular transcutaneous electrical nerve stimulation in depressed patients: a randomized controlled pilot study. J Neur Transm (Vienna). 2013;120(5):821-827.

39. Rong PJ, Fang JL, Wang LP, et al. Transcutaneous vagus nerve stimulation for the treatment of depression: a study protocol for a double blinded randomized clinical trial. BMC Complement Altern Med. 2012;12:255.

40. Berry SM, Broglio K, Bunker M, Jayewardene A, Olin B, Rush AJ. A patient-level meta-analysis of studies evaluating vagus nerve stimulation therapy for treatment-resistant depression. Med Devices (Auckl). 2013;6:17-35.

41. Cimpianu CL, Strube W, Falkai P, Palm U, Hasan A. Vagus nerve stimulation in psychiatry: a systematic review of the available evidence. J Neural Transm (Vienna). 2016;124(1):145-158.

42. Martin J, Martín-Sánchez E. Systematic review and meta-analysis of vagus nerve stimulation in the treatment of depression: variable results based on study designs. Eur Psychiatry. 2012;27(3):147-155.

43. Cleare A, Pariante C, Young A, et al. Evidence-based guidelines for treating depressive disorders with antidepressants: a revision of the 2008 British Association for Psychopharmacology guidelines. J Psychopharmacol. 2015;29(5):459-525.

44. National Institute for Health and Care Excellence. Vagus Nerve Stimulation for Treatment-Resistant Depression. London: NICE; 2009.

45. Milev RV, Giacobbe P, Kennedy SH, et al. Canadian network for mood and anxiety treatments (CANMAT) 2016 clinical guidelines for the management of adults with major depressive disorder - section 4 : neurostimulation treatments. Can J Psychiatry. 2016;61(9):561-575.

46. Rush AJ, Marangell LB, Sackeim HA, et al. Vagus nerve stimulation for treatment-resistant depression: a randomized, controlled acute phase trial. Biol Psychiatry. 2005;58(5):347-354.

47. Rush AJ, Sackeim HA, Marangell LB, et al. Effects of 12 months of vagus nerve stimulation in treatment-resistant depression: a naturalistic study. Biol Psychiatry. 2005;58(5):355-363.

48. Sackeim HA, Brannan SK, Rush AJ, George MS, Marangell LB, Allen J. Durability of antidepressant response to vagus nerve stimulation (VNS). Int J Neuropsychopharmacol. 2007;10(6):817-826.

49. Moher D, Liberati A, Tetzlaff J, Altman DG. Preferred reporting items for systematic reviews and meta-analyses: the PRISMA statement. PLoS Med. 2009;6(7):e1000097.

50. Kraus T, Hösl K, Kiess O, Schanze A, Kornhuber J, Forster C. BOLD fMRI deactivation of limbic and temporal brain structures and mood enhancing effect by transcutaneous vagus nerve stimulation. $J$ Neural Transm (Vienna). 2007;114(11):1485-1493.
51. Trevizol AP, Shiozawa P, Taiar I, et al. Transcutaneous vagus nerve stimulation (taVNS) for major depressive disorder: an open label proofof-concept trial. Brain Stimul. 2016;9(3):453-454.

52. Fang J, Rong P, Hong Y, et al. Transcutaneous vagus nerve stimulation modulates default mode network in major depressive disorder. Biol Psychiatry. 2016;79(4):266-273.

53. Liu J, Fang J, Wang Z, et al. Transcutaneous vagus nerve stimulation modulates amygdala functional connectivity in patients with depression. $J$ Affect Disord. 2016;205:319-326.

54. Möller HJ. Standardized rating scales in psychiatry: methodological basis, their possibilities and limitations and descriptions of important rating scales. World J Biol Psychiatry. 2009;10(1):6-26.

55. Marsland AL, Kuan DC, Sheu LK, et al. Systemic inflammation and resting state connectivity of the default mode network. Brain Behav Immun. 2017;62:162-170.

56. Heaton RK, Clifford DB, Franklin DR, et al. HIV-associated neurocognitive disorders persist in the era of potent antiretroviral therapy: CHARTER study. Neurology. 2010;75(23):2087-2096.

57. Thomas JB, Brier MR, Snyder AZ, Vaida FF, Ances BM. Pathways to neurodegeneration: effects of HIV and aging on resting-state functional connectivity. Neurology. 2013;80(13):1186-1193.

58. Jacobs HI, Riphagen JM, Razat CM, Wiese S, Sack AT. Transcutaneous vagus nerve stimulation boosts associative memory in older individuals. Neurobiol Aging. 2015;36(5):1860-1867.

59. Cohen RA, Seider TR, Navia B. HIV effects on age-associated neurocognitive dysfunction: premature cognitive aging or neurodegenerative disease? Alzheimers Res Ther. 2015;7(1):37.

60. Biggio F, Gorini G, Utzeri C, et al. Chronic vagus nerve stimulation induces neuronal plasticity in the rat hippocampus. Int J Neuropsychopharmacol. 2009;12(9):1209-1221.

61. Furmaga H, Carreno FR, Frazer A. Vagal nerve stimulation rapidly activates brain-derived neurotrophic factor receptor TrkB in rat brain. PLoS One. 2012;7(5): 34844 .

62. Huston JM, Gallowitsch-Puerta M, Ochani M, et al. Transcutaneous vagus nerve stimulation reduces serum high mobility group box 1 levels and improves survival in murine sepsis. Crit Care Med. 2007; 35(12):2762-2768.

63. Lerman I, Hauger R, Sorkin L, et al. Noninvasive transcutaneous vagus nerve stimulation decreases whole blood culture-derived cytokines and chemokines: a randomized, blinded, healthy control pilot trial. Neuromodulation. 2016;19(3):283-290.

64. Ay I, Nasser R, Simon B, Ay H. Transcutaneous cervical vagus nerve stimulation ameliorates acute ischemic injury in rats. Brain Stimul. 2016;9(2):166-173.

65. Ayerbe L, Ayis S, Wolfe CD, Rudd AG. Natural history, predictors and outcomes of depression after stroke: systematic review and metaanalysis. Br J Psychiatry. 2013;202(1):14-21.

66. Hatziioannou T, Evans DT. Animal models for HIV/AIDS research. Nat Rev Microbiol. 2012;10(12):852-867.

67. Tseng A, Szadkowski L, Walmsley S, Salit I, Raboud J. Association of age with polypharmacy and risk of drug interactions with antiretroviral medications in HIV-positive patients. Ann Pharmacother. 2013;47(11):1429-1439.

68. Bet PM, Hugtenburg JG, Penninx BW, Hoogendijk WJ. Side effects of antidepressants during long-term use in a naturalistic setting. Eur Neuropsychopharmacol. 2013;23(11):1443-1451.

69. Casaretti L, Paolillo S, Formisano R, et al. [Metabolic and cardiovascular effects of combined antiretroviral therapy in patients with HIV infection: systematic review of literature]. Monaldi Arch Chest Dis. 2015; 76(4):175-182. Italian.

70. Jernigan MG, Kipp GM, Rather A, Jenkins MT, Chung AM. Clinical implications and management of drug-drug interactions between antiretroviral agents and psychotropic medications. Ment Health Clin. 2013; 2(9):274-285. 


\section{Publish your work in this journal}

Neuropsychiatric Disease and Treatment is an international, peerreviewed journal of clinical therapeutics and pharmacology focusing on concise rapid reporting of clinical or pre-clinical studies on a range of neuropsychiatric and neurological disorders. This journal is indexed on PubMed Central, the 'PsycINFO' database and CAS, and is the official journal of The International Neuropsychiatric Association (INA). The manuscript management system is completely online and includes a very quick and fair peer-review system, which is all easy to use. Visit http://www.dovepress.com/testimonials.php to read real quotes from published authors.

Submit your manuscript here: http://www.dovepress.com/neuropsychiatric-disease-and-treatment-journal 